\title{
Galactic Cosmic Ray modulation based on transport parameters obtained via test particle simulations
}

\author{
J. F. Valdés-Galicia ${ }^{1}$, R. A. Caballero-López ${ }^{1}$, and H. Moraal ${ }^{2}$ \\ 1 Instituto de Geofísica, UNAM, 04510 México, D.F., Mexico \\ e-mail: [jfvaldes;rogelioc]@geofisica.unam.mx \\ 2 School of Physics, North-West University, Potchefstroom 2520, South Africa \\ e-mail: FSKHM@puknet.puk.ac.za \\ Received 30 January 2006 / accepted 21 May 2006
}

\section{ABSTRACT}

\begin{abstract}
Our understanding of Galactic Cosmic Ray (GCR) modulation has advanced greatly in the last years. However, an appropriate knowledge of the components of the diffusion tensor is still lacking. Numerical simulations of test particle trajectories in the layer model of the Heliospheric Magnetic Field allow calculations of local transport coefficients. The layer model makes use of spacecraft data at different heliospheric locations to reproduce transport conditions; there are no special assumptions as to the form of the turbulence. We use these test particle estimated transport coefficients here as parameters for a numerical solution of the GCR transport equation in the case $q A>0$. The solutions obtained with this set of parameters reproduce the observed cosmic ray proton spectrum at $1 \mathrm{AU}$ and the radial profiles up to at least $20 \mathrm{AU}$ in the ecliptic plane well.
\end{abstract}

Key words. interplanetary medium - Sun: magnetic fields - convection - diffusion

\section{Introduction}

The Galactic Cosmic Ray (GCR) transport equation to study solar modulation in the heliosphere includes the effects of solar wind convection, adiabatic deceleration due to the solar wind expansion, and the diffusion produced by the Heliospheric Magnetic Field (HMF) structure and turbulence. To solve the GCR transport equation, it is essential to have appropriate knowledge of the parameters of the diffusion tensor, K. The different coefficients of $\mathrm{K}$ describe diffusion parallel and perpendicular to the HMF, as well as the effects of drifts caused by the field gradient, curvature, and the heliospheric neutral sheet. The form and magnitude of the drift effects are reasonably well understood (see, e.g., Munakata 2003); however, there are still efforts to be made to understand the physical mechanisms leading to parallel and perpendicular diffusion, since they are closely related to the solar wind turbulence whose comprehension is still not complete (see, e.g., Parhit et al. 2003; De Koning \& Bieber 2003).

The approach we take here to solve the GCR transport equation does not make any special assumption or rely on any theory regarding the form or structure of the turbulence. We use parallel and perpendicular diffusion coefficients obtained with a numerical model to follow test particle trajectories. The model makes use of high time resolution spacecraft HMF measurements at different locations in the ecliptic plane of the heliosphere, ranging from 1 to 20 AU (see Valdés-Galicia 1993, and references therein). In principle, all the local fluctuations are automatically incorporated in these simulations, and their effects will be sensed as the test particles propagate in the layer model of the HMF that will be described in the next section.

\section{Model and parameters}

With the aim of reproducing observations of spacecrafts at different heliospheric locations, Steenkamp (1995) and
Steenberg (1998) developed a numerical scheme to solve the GCR transport equation, $f(\boldsymbol{r}, p, t)$ :

$\partial f / \partial t+\boldsymbol{V} \cdot \nabla f-\nabla(\mathrm{K} \cdot \nabla f)-(1 / 3)(\nabla \cdot \boldsymbol{V}) \partial f / \partial \ln p=Q$

where $p$ is particle momentum, $\boldsymbol{V}$ is the solar wind velocity, and $\mathrm{K}$ is the diffusion tensor that includes parallel and perpendicular diffusion, as well as gradient and curvature drifts. This solution has been successful in reproducing several aspects of the observed cosmic ray phenomenology inside the heliosphere (see, e.g., Steenberg et al. 1999; Caballero-Lopez et al. 2004). As was already pointed out, our aim here is to test the solution making use of numerically calculated transport parameters.

As indicated by Ulysses observations, we have used a solar wind velocity of $400 \mathrm{~km} \mathrm{~s}^{-1}$ in the ecliptic regions, increasing between $10^{\circ}$ and $30^{\circ}$ latitude to $800 \mathrm{~km} \mathrm{~s}^{-1}$ and constant thereafter (McComas 2003). The heliospheric boundary was set at $90 \mathrm{AU}$. We have solved Eq. (1) only for proton species and for the case $q A>0$. At the heliospheric boundary, we impose the local interstellar proton spectrum (LIS) estimated by Webber and Lockwood (2001), as shown in Fig. 3. The HMF used is a modified Parker spiral field, as given by Caballero-Lopez et al. (2004). The main goal of this work is to use the transport parameters, obtained from the layer model between 1 to $20 \mathrm{AU}$, to analyse cosmic ray modulation in this region of the heliosphere. According to Caballero-Lopez et al. (2004), the effects of the shock acceleration and heliosheath modulation are small in the inner heliosphere, due to the strong adiabatic energy losses over the supersonic solar wind region inside the shock (see their Fig. 6, panel B).

The remaining parameters for the solution are those of the diffusion tensor. The set used here are the results of test particle numerical simulations in an HMF model. The layer model of the HMF makes use of magnetometer spacecraft measurements. The main assumption of this model is that the field fluctuations seen along a spacecraft trajectory are, on a statistical average, 


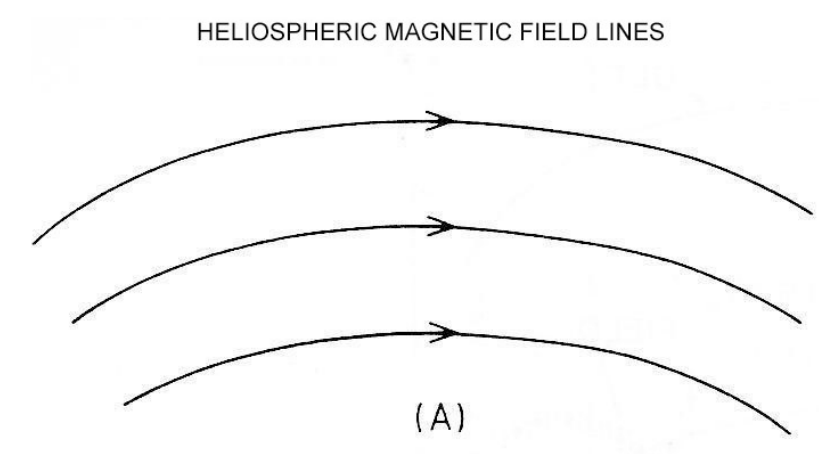

(A)

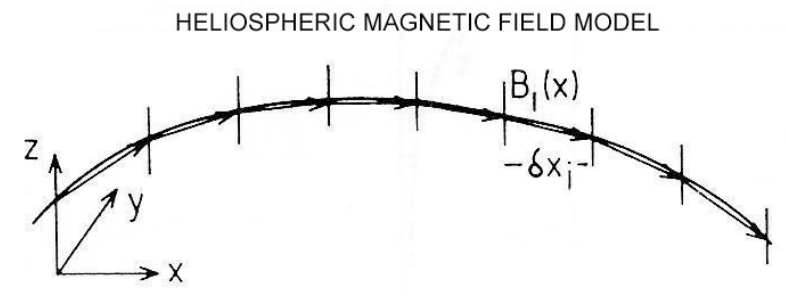

(B)

Fig. 1. Schematic representation of the layer model of the HMF (see text).

of the same sort as those that a particle would encounter as it propagates along a field line. This being so, resonant scattering, asymmetric distribution of wave-vectors, mirroring near $90^{\circ}$, non-helical trajectories, or specific HMF helicity are all automatically taken into account. Each of the layers has a width $\left(\delta x_{\mathrm{i}}\right)$ given by the sampling time multiplied by the solar wind speed $\left(\delta x_{\mathrm{i}}=\boldsymbol{V} \times \delta \tau\right)$. Thus, a HMF line is approximated by a polygonal line that consists of consecutive vectors of the magnetic field. The layer must be thin compared with the particle gyroradius, as it determines the integration time step (see Fig. 1). Transport coefficients for any component of the phase space can be calculated following a large number of test particle trajectories. For more details of the model see, e.g., Moussas et al. (1982) or Valdés-Galicia (1993).

Test particle simulations using data from various spacecraft located between 1 to $20 \mathrm{AU}$ were performed to calculate the parallel, $\kappa_{\|}$, and perpendicular, $\kappa_{\perp}$, diffusion coefficients. The radial dependence of these coefficients was estimated to be roughly proportional to $r^{0.3}$ (Moussas et al. 1992; Valdés-Galicia et al. 1992). These results allowed us to set:

$\kappa_{r r}=[4.6 \beta P(G V) f(r) g(\theta)] 6 \times 10^{20} \mathrm{~cm}^{2} / \mathrm{s}$,

where $\kappa_{r r}=\kappa_{\|} \cos ^{2} \psi+\kappa_{\perp} \sin ^{2} \psi, \beta$ is particle speed in terms of the speed of light, and $f(r)=r^{0.3}$ for $r<30 \mathrm{AU}$, extrapolated as $f(r)=$ constant for $r>30$ AU. Test particle determined diffusion coefficients exist only in the ecliptic plane; therefore, we choose $g(\theta)=(5+3 \cos (2 \theta)) / 2$. This is a symmetric function in $\theta$ that makes the diffusion coefficients diminish with heliolatitude to represent the presence of a more turbulent region close to the equator. Additionally $\kappa_{r r}=\kappa_{\theta \theta}$ throughout.

Mean free paths $\lambda=3 \kappa / v$ are calculated from the parallel and perpendicular coefficients, as $\lambda_{r r}=\lambda_{\|} \cos ^{2} \psi+\lambda_{\perp} \sin ^{2} \psi$ and $\lambda_{r r}=\lambda_{\theta \theta}$. The results are plotted in Fig. 2. The most noteworthy facts from the plots are the growth of $\lambda_{r r}$ and $\lambda_{\theta \theta}$ with radial distance, as determined by the test particle numerical experiments and its inverse dependence on polar angle. These
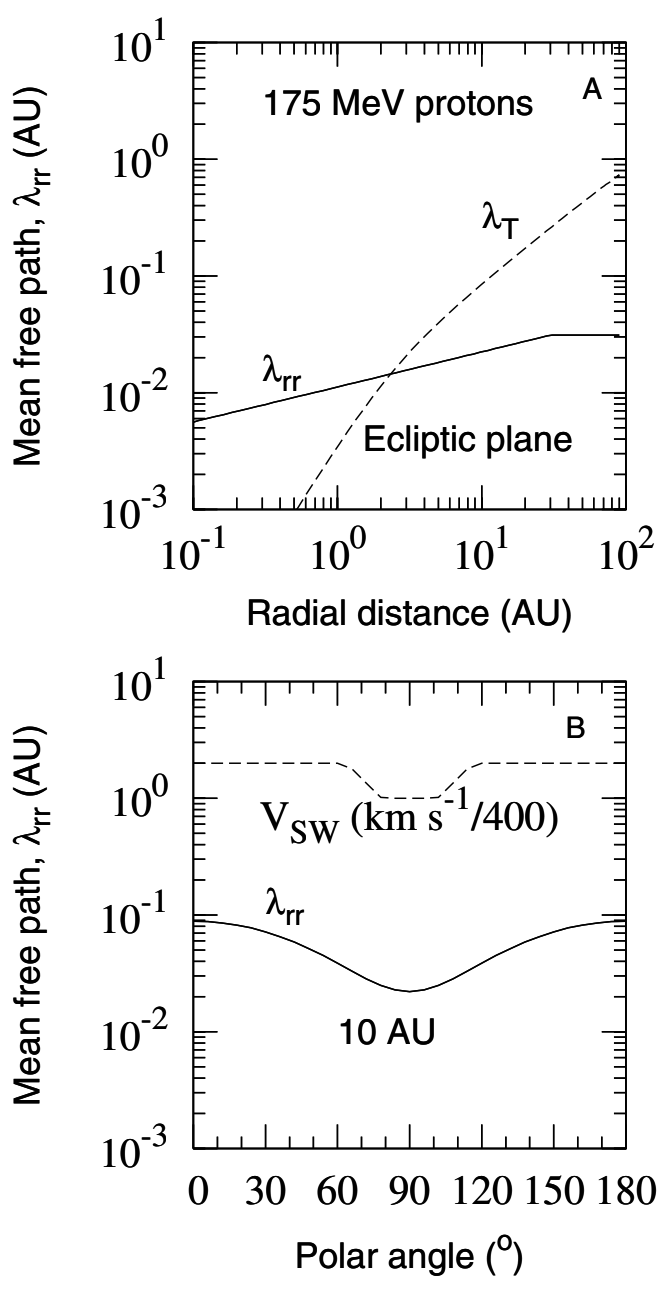

Fig. 2. A) Radial dependence of the mean free paths used in the solution of the transport equation. B) Latitudinal dependence of the solar wind velocity and the radial mean free path.

functional forms have successfully been tried in the past with the present solution of Eq. (1).

The asymmetric coefficient of the diffusion tensor is calculated as $\kappa_{\mathrm{T}}=\beta P /(3 B)$, describing the HMF gradient and curvature drifts. The drift velocity is $\boldsymbol{v}_{\mathrm{d}}=(\beta P / 3) \nabla \times \boldsymbol{B} / B^{2}$. Figure $2 \mathrm{~A}$ shows that $\lambda_{\mathrm{T}}$ is larger than the diffusion mean free paths $\lambda_{r r}$ and $\lambda_{\theta \theta}$ from $\sim 2 \mathrm{AU}$ outward, and therefore this is a drift dominated model.

The wavy neutral sheet tilt angle is set at $10^{\circ}$, while the neutral sheet drift is handled by the method of Steenberg (1998). The initial condition of the solution is that the LIS pervades the entire heliosphere, and it is stepped forward in time until it reaches steady state.

\section{Results and discussion}

Proton spectra of the balloon-borne BESS experiment registered in July 1997 (Myers et al. 2003) and IMP8 spacecraft observations from April 20, 1997 to March 23, 1998 are shown in Fig. 3. The proton intensity spectrum as obtained from the solution of Eq. (1) at the Earth's orbit, using the set of parameters presented in Sect. 2 of this paper, as well as the LIS, are also shown. Observations correspond to a period around the minimum of cycle 22, when $q A>0$. The BESS data serve as a good reference since they have high accuracy and cover a wide energy 


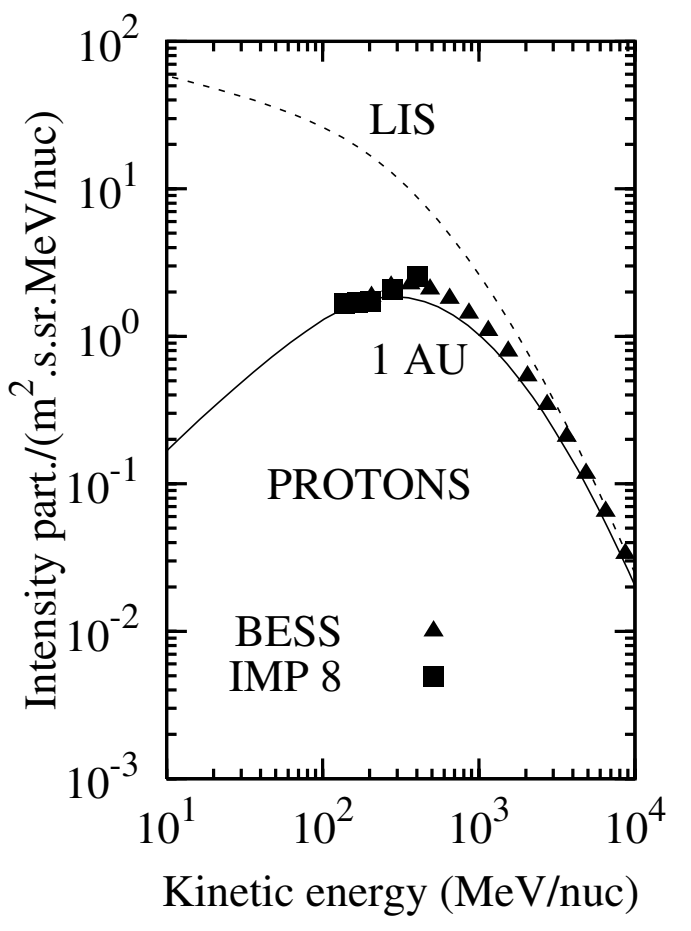

Fig. 3. The proton spectrum obtained in this work at $1 \mathrm{AU}$ compared with data from IMP 8 and BESS. We used the local interstellar medium spectrum (LIS) of Webber \& Lockwood (2001) as a boundary condition.

range, providing excellent information about the rigidity dependence of the modulation. The solution fits the observations well from $10^{2}$ to $10^{4} \mathrm{MeV}$, which shows that the set of transport parameters used in the solution is able to reproduce features of the solar modulation of GCR. Therefore, the turbulence present in the layer model of the HMF used to perform test particle numerical experiments is a realistic representation of the conditions found by GCR as they propagate into the heliosphere. In particular, the significant growth of the mean free path from the inner heliosphere out to around 30 AU reveals the diminishing ability of the measured HMF irregularities to scatter particles as the distance from the sun increases.

Cosmic ray radial intensity profiles obtained from the solution of Eq. (1) are presented in Fig. 4. Protons with an average kinetic energy of $175 \mathrm{MeV}$ were used, i.e., in the range of energies of the test particle simulations. The curves shown correspond to intensities in the ecliptic plane, at $\theta=60^{\circ}$ (latitude $30^{\circ}$ ), at $\theta=30^{\circ}$ (latitude $60^{\circ}$ ), and at the pole. The intensities are normalized at the boundary, set at 90 AU. Squares correspond to observations by several spacecraft: IMP8 at 1 AU, Voyager 2 at $1.8 \mathrm{AU}$, Voyager 1 at $2 \mathrm{AU}$, Pioneer 11 at $7 \mathrm{AU}, 15^{\circ} \mathrm{N}$, and Pioneer 10 at $12 \mathrm{AU}$ in $1977(q A>0)$. The outermost two data points are from Voyager 2 at $56 \mathrm{AU}, 24^{\circ} \mathrm{S}$ and Voyager 1 at $72 \mathrm{AU}, 34^{\circ} \mathrm{N}$, in 1997 . Combining data from the two minima at 1977 and 1997 is justified by observations (McDonald et al. 2001).

The range of heliodistances covered by the test particle simulations goes from 1 to $20 \mathrm{AU}$. Within this region, the radial profile produced by the solution in the ecliptic plane fits all the observations well. Voyager 1 and 2 were $34^{\circ} \mathrm{N}$ and $24^{\circ} \mathrm{S}$ of the ecliptic plane in 1997 when the data corresponding to the points at 72 and $56 \mathrm{AU}$ were taken. They should therefore correspond with the dashed curve representing intensities at a polar angle of $60^{\circ}$ and not with the ecliptic plane curve. This implies that the

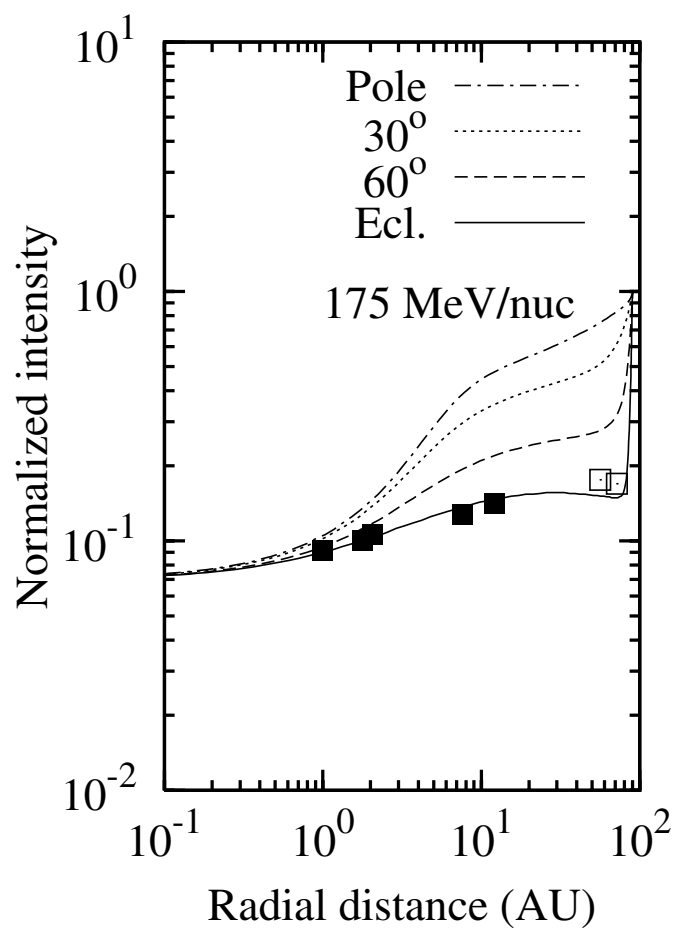

Fig. 4. Radial profiles of the solutions obtained from this work. Full squares (from 1 to $12 \mathrm{AU}$ ) correspond to spacecraft measurements in the ecliptic plane during solar minimum periods when $q A>0$. The outermost two (open) squares at 56 and $72 \mathrm{AU}$ correspond to data taken at latitudes far from the ecliptic plane (see text).

latitudinal gradients produced by this solution are much higher than observed. However, a further increase in the cross field diffusion beyond $30 \mathrm{AU}$ could produce the reduced cosmic ray gradients calculated from spacecraft counting rates in that region. The intensity curves predicted by the present solution in the outermost portion of the heliosphere are perhaps too steep, as they would imply a substantial reduction of the GCR flux in a thin region close to the boundary. Test particle simulations with HMF data taken in the outer heliosphere seem to be necessary to answer the questions posed by the results of this paper.

The test particle simulations were done with data from spacecraft in the ecliptic plane, with a very localized validity. It is therefore encouraging to see that the calculated transport parameters used in solutions of Eq. (1) are able to reproduce observations in the range of heliospheric locations where the simulations were done. This is a further proof that the layer model of the HMF is able to adequately represent the local turbulence found by charged energetic particles and can be used to calculate parameters that represent the transport conditions to which GCR are subject.

The layer model of the HMF was also successful in estimating representative mean free paths calculated with data from the Helios spacecraft in the inner heliosphere. Several solar proton events's time profiles and anisotropies with different levels of scattering were appropriately reproduced (Valdés-Galicia et al. 1995).

Here we present, results of the solution of Eq. (1) for the case when $q A>0$, when positively charged particles drift from the poles to the ecliptic. We have not yet been able to obtain solutions that reproduce observations in the other half of the solar magnetic cycle $(q A<0)$. 


\section{Summary and conclusions}

High time resolution magnetometer data from spacecraft located in the ecliptic plane from 1 to $20 \mathrm{AU}$ were used in the layer model of the HMF to calculate test particle transport parameters. In this work, we used parallel and perpendicular diffusion coefficients calculated with the layer model as input parameters in a solution of the GCR transport equation.

Solutions for protons at solar minimum conditions for $q A>$ 0 reproduce the observed spectrum at $1 \mathrm{AU}$ in the range from $10^{2}$ to $10^{4} \mathrm{MeV} /$ nuc well. The measured cosmic ray radial profiles in the ecliptic plane out to about $20 \mathrm{AU}$ are also reasonably well fitted by our solutions. The latitudinal and radial gradients in the outermost heliosphere obtained do not reproduce observations. In these regions, assumptions were made as to the transport conditions, since there are no test particle determined transport parameters further than $20 \mathrm{AU}$ from the Sun.

Acknowledgements. R.C.L. was supported by UNAM-DGAPA grants IN106105. JFVG received partial support from CONACYT grant 40601 and DGAPA-UNAM 116503. H.M. is supported by the South African National Research Foundation.

\section{References}

Caballero-Lopez, R. A., Moraal, H., \& McDonald, F. B. 2004, J. Geophys. Res., A05105

De Koning, C. A., \& Bieber, J. W. 2003, Proc. 28 ICRC, Tsukuba, 6, 3717

McComas, D. J., Elliott, H. A., Schwadron, N. A., et al. 2003, Geophys. Res. Lett., 30, 1517

McDonald, F. B., Cummings, A. C., Lal, N., et al. 2003, Proc. 27 ICRC, Hamburg, 9, 3830

Moussas, X., Quenby, J. J., \& Valdés-Galicia, J. F. 1982, Ap\&SS, 85, 99

Moussas, X., Quenby, J. J., Theodosiu, Z., et al. 1992, Sol. Phys., 140, 161

Munakata, K. 2003, Proc. 28 ICRC, Tsukuba, 8, 251

Myers, Z. D., Seo, E. S., Abe, K., et al. 2003, Proc. 28 ICRC, Tsukuba, 4, 1805

Parhi, S., Bieber, J. W., Matthaeus, W. H., \& Burger, R. A. 2003, AJ, 585, 502

Steenberg, C. D. 1998, Ph.D. Thesis, Potchefstroom University, South Africa

Steenberg, C. D., Cummings, A. C., \& Stone, E. C. 1999, Proc. 26 ICRC, Salt Lake City, 7, 593

Steenkamp, R. 1995, Ph.D. Thesis, Potchefstroom University, South Africa

Valdés-Galicia, J. F. 1993, Space Sci. Rev., 62, 67

Valdés-Galicia, J. F., Quenby, J. J., \& Moussas, X. 1992, Sol. Phys., 139, 189

Valdés-Galicia, J. F., Wanner, W., Kallenrode, M. B., \& Wibberenz, G. 1995, ApJ, 439, 482

Webber, W. R., \& Lockwood, J. A. 2001, J. Geophys. Res., 106, 29323 